\title{
Buenas prácticas de producción de cacao en comunidades víctimas del conflicto, Granada (Colombia)
}

\section{Good practices of cocoa production in communities victims of the conflict, Granada (Colombia)}

\section{Boas práticas de produção de cacau em comunidades vítimas do conflito,}

\section{Granada (Colômbia)}

\author{
Rodriguez Guevara Jessica Naiyuri ${ }^{1}$ y María Cristina Ospina Ladino² \\ ${ }^{1}$ Ingeniera Agroindustrial Universidad de los Llanos y \\ ${ }^{2}$ Ingeniera Agroindustrial, Docente Universidad de los Llanos \\ jessica.rodriguez.guevara@unillanos.edu.co
}

Recibido 31 de octubre 2019, Aceptado 12 de marzo 2020

\section{RESUMEN}

El objetivo de este proyecto estuvo encaminado a poder contribuir como institución en el progreso de las comunidades víctimas del conflicto armado, que en décadas pasadas fueron obligadas a participar en la producción de cultivos ilícitos debido a las limitadas alternativas de ingreso en la zona; por ello el desarrollo de este proyecto tuvo lugar en la zona del medio Ariari, en el municipio de Granada del departamento del Meta donde la población beneficiaria tiene cultivo de cacao que no superan las cinco hectáreas por productor. Por lo anterior, se capacitaron a 50 personas de poblaciones víctimas del conflicto, campesinos y mujeres; en procesos como beneficio y transformación del cacao, buscando el fortalecimiento de esta cadena productiva debido a que es un producto apetecido para industrias de chocolates a nivel y el mercado exterior. Se diseñó una capacitación llamada "procesos productivos del cacao" que incluyó etapas desde la recolección de las mazorcas hasta la obtención de diferentes productos agroindustriales que generan ingresos a las familias campesinas y dinamizan la economía del sector. Esta capacitación fue subdividida en 6 subconferencias: Beneficio del cacao, transformación del cacao en chocolate de mesa, instalaciones de una planta de transformación de cacao, equipos necesarios en la transformación de cacao, 
obtención del licor de cacao y alternativas de agroindustrialización, donde se obtuvo una participación activa por parte de los cincuenta productores asistentes, gran aceptación y disposición en las temáticas impartidas, lo cual muestra el interés que genera este tipo de actividades para las poblaciones que contribuyen al desarrollo de la región.

Palabras clave: Ariari Colombia, cacao, conflicto armado, agroindustria.

\section{ABSTRACT}

The objective of this project was aimed at being able to contribute as an institution to the progress of the communities victims of the armed conflict, which in past decades were forced to participate in the production of illicit crops due to the limited income alternatives in the area; for this reason, the development of this project took place in the middle Ariari area, in the Granada municipality of the Meta department, where the beneficiary population has cocoa cultivation that does not exceed five hectares per producer. Therefore, 50 people from populations victims of the conflict, peasants and women were trained; in processes such as processing and transformation of cocoa, seeking to strengthen this production chain because it is a desired product for chocolate industries at the level and the foreign market. A training called "cocoa production processes" was designed, which included stages from harvesting the pods to obtaining different agro-industrial products that generate income for farm families and boost the economy of the sector. This training was subdivided into 6 sub-conferences: Cocoa processing, transformation of cocoa into table chocolate, facilities of a cocoa transformation plant, equipment necessary for cocoa transformation, obtaining of cocoa liquor and agroindustrialization alternatives, where it was obtained active participation by the fifty attending producers, great acceptance and willingness in the topics taught, which shows the interest that this type of activity generates for the populations that contribute to the development of the region.

Keywords: Ariari Colombia, cocoa, armed conflict, agro-industry. 


\section{RESUMO}

O objetivo deste projeto era poder contribuir como instituição para o progresso das comunidades vítimas do conflito armado, que nas últimas décadas foram forçadas a participar da produção de cultivos ilícitos devido às alternativas de renda limitada na área; por esse motivo, o desenvolvimento deste projeto ocorreu na área central de Ariari, no município de Granada, no departamento de Meta, onde a população beneficiária possui cultivo de cacau que não excede cinco hectares por produtor. Portanto, 50 pessoas de populações vítimas do conflito, camponeses e mulheres foram treinadas; em processos como processamento e transformação de cacau, buscando fortalecer essa cadeia produtiva por ser um produto desejado para as indústrias de chocolate no nível e no mercado externo. Foi desenvolvido um treinamento chamado "processos de produção de cacau", que incluía etapas desde a colheita das vagens até a obtenção de diferentes produtos agroindustriais que geram renda para as famílias de agricultores e impulsionam a economia do setor.

Este treinamento foi subdividido em 6 subconferências: processamento de cacau, transformação de cacau em chocolate de mesa, instalações de uma planta de transformação de cacau, equipamento necessário na transformação de cacau, obtenção de alternativas de licor de cacau e agroindustrialização, onde foram obtidos participação ativa dos cinquenta produtores presentes, grande aceitação e disposição nas disciplinas ministradas, o que mostra o interesse que esse tipo de atividade gera para as populações que contribuem para o desenvolvimento da região.

Palavras-chave: Ariari Colômbia, cacau, conflito armado, agroindústria.

\section{INTRODUCCIÓN}

El cultivo de cacao en Colombia no ha sido fácil de consolidar, debido a la que las zonas productoras han estado involucradas en el conflicto armado: Huila, Santander, Arauca, Urabá Antioqueño y Meta (Gómez y García, 2016). Colombia se ha caracterizado por sufrir violencia, proveniente de varios grupos ilegales, finalmente en el 2016 se dio un gran paso al firmar un acuerdo de paz con el grupo 
guerrillero de las FARC (Fuerzas Armadas Revolucionarias de Colombia), con quien se sostuvo un conflicto que duró más de 50 años, perjudicando durante este tiempo al país y su población en todos los municipios aislados, entre los que se encuentra Granada.

\section{COMUNIDADES VÍCTIMAS}

En Colombia de acuerdo a la Ley 1448 de 2011, se establece que las víctimas son: "Aquellas personas que individual o colectivamente hayan sufrido un daño por hechos ocurridos a partir del $1^{\circ}$ de enero de 1985 , como consecuencia de infracciones al Derecho Internacional Humanitario o de violaciones graves y manifiestas a las normas internacionales de Derechos Humanos, ocurridas con ocasión del conflicto armado interno. El cónyuge, compañero o compañera permanente, parejas del mismo sexo y familiar en primer grado de consanguinidad, primero civil de la víctima directa, cuando a esta se le hubiere dado muerte o estuviere desaparecida. A falta de estas, lo serán los que se encuentren en el segundo grado de consanguinidad ascendente. De la misma forma, a las personas que hayan sufrido un daño al intervenir para asistir a la víctima en peligro o para prevenir la victimización. La condición de víctima se adquiere con independencia de que se individualice, aprehenda, procese o condene al autor de la conducta punible y de la relación que pueda existir entre el autor y la víctima" (CRC, 2011).

Gracias a la firma del acuerdo de paz en Colombia la producción de cacao aumentó, tanto así que el mercado extranjero demostró estar interesado ya no solo por la producción de café si no por las características deseables presentes en el grano de cacao. Según la Federación nacional de cacaoteros (FEDECACAO, 2018), hace diez años el país exportaba 650 toneladas, en el año 2017 el presidente de esta federación afirma que las ventas externas alcanzaron 11.926 toneladas en grano y en el 2016 el país logró su mayor producción de cacao con 60.000 toneladas.

Lo anterior demuestra el potencial cacaotero del país, no solo en la producción del grano de cacao seco sino en la participación de productos de exportación con valor agregado, las asociaciones en articulación con FEDECACAO, Alcaldías, 
Universidades Públicas y Gobierno Nacional con la participación de los Ministerios de Educación y Agricultura, han venido beneficiando a los productores de cacao con capacitaciones que abarcan los procesos de cultivo, enfermedades, beneficio y transformación, que permitirán estandarizar los procesos y competir con productos ya establecidos en el mercado nacional e internacional.

Según Ramírez y Jaramillo, (2014) Colombia se encuentra ubicada en la zona intertropical, por lo tanto durante todo el año no presenta variaciones de temperatura significativas, lo que favorece la producción de algunos cultivos, sumado a la gran porción de selva amazónica que posee, le ha permitido posicionarse como un territorio mega diverso en flora y fauna a nivel mundial caracterizándose por ser un país que basa su economía en la agricultura. Es importante resaltar que el cacao producido en Colombia según la organización mundial de cacao (Loureiro et al., 2017). Este producto está catalogado de calidad por su sabor fino y exquisito aroma debido a la calidad de los genotipos que tienen un mercado especial para la comercialización de productos diferenciados por su calidad y variedad. Razón por la cual impulsar su producción resulta ser una oportunidad para el desarrollo del departamento del Meta, buscando que a través del conocimiento transmitido a

productores y líderes de asociaciones se pueda aplicar e incentivar al productor a realizar buenas prácticas de cosecha y pos cosecha y que se anime a realizar la transformación del producto siempre cumpliendo con las normas de sanidad y calidad, ya que si se dinamiza la cadena cacao-chocolate esto ayudaría a aumentar los ingresos de las familias productoras.

\section{SITUACIÓN DEL CULTIVO DEL CACAO}

Según Argout et al., 2011, el cacao (Theobroma cacao L.) es una planta de hoja perenne, que se cultiva en los países están en la zona intertropical (Figura 1), siendo el tercer producto más importante de exportación agrícola en el mundo (sólo superado por el café y el azúcar) y que además provee beneficios económicos a algunas zonas pobres del mundo, en especial a aquellos países como Costa de Marfil que dominan la producción mundial. Además de servir para obtener el chocolate, Según Osorio et al., (2017), los granos de cacao también se utilizan para 
la fabricación de dulces, jaleas, helados, licores, productos cosméticos y medicinales. Son tres tipos de Cacao, los más comercializados a nivel mundial: forastero, criollo y trinitario, los tres se cultivan en Colombia.

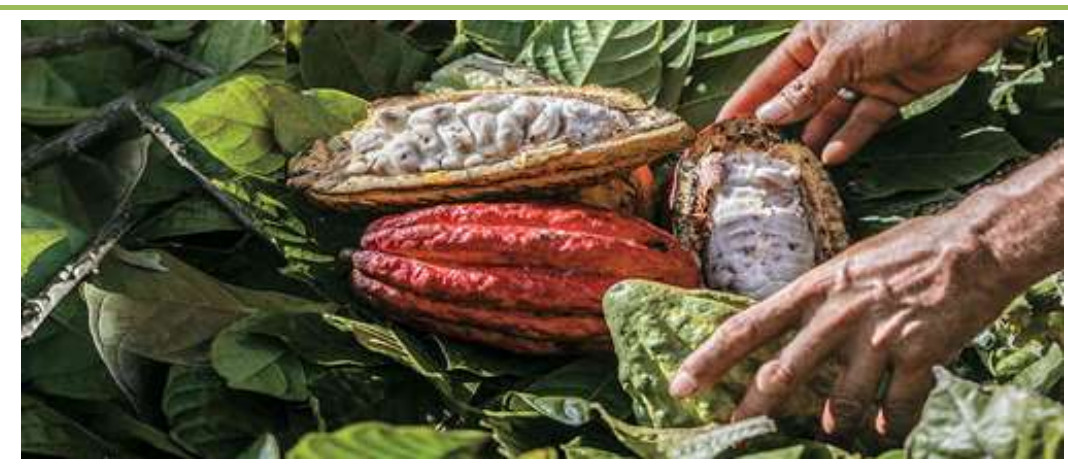

Figura 1. Cultivo de cacao alternativa productiva y social Fuente: Acosta y Martinez, (2017).

Según las cifras de DANE, (2017) en Colombia el área cultivada de cacao para el fue para este año 160.276 ha con una producción aproximada de 47.732 toneladas de grano, posicionándose así como el décimo país y área productora cosechada en el mundo, así como el tercero en América Latina por detrás de Brasil y Ecuador. Desde 1960, para Osorio et al., (2017), la producción colombiana de cacao no ha mejorado considerablemente, debido en parte a la incidencia de enfermedades y la plantación monoclonal, para lo cual se han creado bancos de germoplasma Ex situ con el objetivo de apoyar la supervivencia de la especie en su hábitat natural y para conservar diversidad de especies para ser utilizadas en programas de mejoramiento.

El cultivo de cacao se cultiva en climas cálidos y húmedos, a temperaturas entre 25 a $35^{\circ} \mathrm{C}$, precipitaciones de 1000 a $3000 \mathrm{~mm} /$ año, los suelos franco arcillosos con buen drenaje y $\mathrm{pH}$ de 4 a 7,4. Sus frutos son en forma de bayas alargadas llegando a pesar un $\mathrm{kg}$, su pulpa contiene de 20 a 40 semillas de color variable entre crema, morado, rojizo o café. El control de plagas y enfermedades se recomienda hacerlo desde el vivero hasta su cosecha, para lo cual se emplean métodos físicos, biológicos, químicos y culturales para mitigar la problemática fitosanitaria causada por estos agentes, los insectos que se encuentran con mayor frecuencia son: 
Coleópteros, Homópteros y Lepidópteros, otras enfermedades de importancia son: moniliasis, Escoba de Bruja y Fitóftora (Rojas y Sacristán, 2013).

La forma más recomendada de realizar su siembra es por medio de propagación por injerto, teniendo en cuenta que se puede dar en campo y vivero, a la hora de llevarla a campo es recomendable que sea en periodos de lluvias ya que así se logra una mayor producción, un inicio de cosecha en menor tiempo y una mejor calidad de producto, con una distancia de siembra recomendada de $3 \times 3$ metros en cuadro o triangulo para densidades que oscilen entre 1100 y 1280 plantas por hectáreas, para densidades más bajas como de 1000 árboles por hectáreas se puede utilizar una distancia de 3,3 x 3,3 metros (Arévalo et al., 2017).

Por el aroma y sabor que tienen los granos de cacao, ha conllevado que con este producto se elaboren derivados, que tienen una buena aceptación en el mercado alimentario (bebidas instantáneas y con grado de alcohol, queso con cubierta de chocolate y dulces) farmacéutico y cosmético (extracción de polifenoles, jabones); siendo estos productos de fácil preparación y con bajo costo de inversión. Según el análisis, se ha visto reflejando el interés de los países no productores en el desarrollo e innovación de productos. El crecimiento ha sido progresivo, lo que sugiere enfoques estratégicos para patentar, lo cual está actualmente dominado por empresas europeas y asiáticas. Comprendiendo estas tendencias mundiales, permitirá desarrollar capacidades con la generación de nuevos productos orientados a emprender agroindustrias dando oportunidad socioeconómica en estas zonas, afectadas por violencia (Gómez et al., 2019)

En Colombia se han realizado adelantos en investigaciones, trabajos y proyectos integrando a la población víctima de los flagelos de la guerra con el fin de mejorar economía, unión social y empoderamiento personal buscando cadenas sustentables, representando en este caso la cadena productiva del cacao. Se han planteado estrategias para aprovechar el cacao en tiempo de posconflicto apoyándose en el Acuerdo de integración Colombia-Unión Europea, donde primero se realizó un análisis para conocer la producción y comercialización que tiene a 
escala mundial, identificar y diagnosticar los problemas que tiene su producción y comercialización en Colombia (Cediel, 2017).

Es importante tener en cuenta que las exportaciones de cacao han aumentado reflejándose la elevada demanda extranjera y esto es positivo para los productores nacionales, puesto que se mejora la calidad de vida de productores, ofreciendo. Oportunidades y ventajas comparativa que posee el cacao colombiano en la fase del posconflicto (Cristancho et al., 2017).

El presente proyecto, se desarrolló en el marco del convenio interadministrativo 833 entre el Ministerio de Educación (MEN) y la Universidad de los Llanos, cuyo objeto es "Aunar esfuerzos entre el Ministerio de Educación (MEN) y la Universidad de los Llanos para promover el Desarrollo Rural a través del Plan Estratégico de Educación Rural en el Departamento del Meta denominado "Ética Ambiental y Bienestar Animal: Estrategia de Desarrollo Rural en Paz", firmado el 26 de enero de 2018 (UNILLANOS, 2018).

\section{METODOLOGÍA}

\section{Ubicación}

Este trabajo se realizó en el municipio de Granada, ubicado al oriente de Colombia en el departamento del Meta; el área municipal es de $350 \mathrm{~km}^{2}$ y el número de habitantes de 98.500. El municipio se encuentra entre los $3^{\circ} 26^{\prime \prime}$ de latitud norte y $73^{\circ} 43^{\prime \prime}$ de longitud oeste, y desde 372 a 410 metros sobre el nivel del mar. Los veranos son cortos, muy calientes, húmedos y nublados; los inviernos son cortos, cálidos y parcialmente nublados y está mojado durante todo el año. Durante el transcurso del año, la temperatura generalmente varía de 19 a $31^{\circ} \mathrm{C}$ y rara vez baja a menos de $15^{\circ} \mathrm{C}$ o sube a más de $34^{\circ} \mathrm{C}$.

\section{Recolección de información y capacitación}

El presente trabajo tuvo por objetivo capacitar a 50 personas del municipio de Granda (Meta), pequeños productores de cacao de las asociaciones ASOPCARIAGAMETA y no asociados, víctimas del conflicto armado, mujeres y 
afrodescendientes, fortaleciendo las buenas prácticas sanitarias en estos sistemas productivos que fueron evaluados de forma cualitativa por medio de encuestas. Se llevó a cabo el reconocimiento de esta población a capacitar, a quienes se les aplicó una encuesta, la cual se dividió en dos secciones, la primera compuesta por ocho preguntas que hacen referencia a puntos generales como el tipo de población, sexo, si cuenta con plantación de cacao, ya había tomado o realizado curso de cacao al igual que algún proceso que interviene en la cadena productiva del cacao. La segunda consta de once preguntas de conocimientos puntuales sobre los procesos, reacciones, condiciones y generalidades que se presentan desde la cosecha hasta la transformación del cacao, ésta segunda parte se aplicó al inicio y al final para evaluar los conocimientos adquiridos.

\section{Etapas del proyecto}

Para el presente proyecto se contemplaron, tres etapas: en la primera se recolectó información acerca de la actividad agrícola y pecuaria a la que se dedican los beneficiarios, además de determinar la participación en cultivos de cacao ya establecidos. El instrumento base utilizado fue una encuesta donde se resaltaron preguntas generales como: edad, sexo, población, tipo de cultivo producido en la región, conocimiento en los procesos de fermentación, secado y obtención de subproductos de cacao (Figura 2).

En la segunda etapa se aplicó una encuesta con 11 preguntas de conocimiento, en donde se evaluaron los procesos de beneficio y transformación del cacao. Luego de recabar la información general, de conocimiento y la participación agrícola y pecuaria de los 50 beneficiarios, se diseñaron las seis (6) guías para las seis conferencias: Beneficio del cacao, transformación, instalaciones de plantas de transformación, equipos utilizados en la transformación de cacao y alternativas de agroindustrialización, además se preparó material audiovisual para la explicación de cada una de las conferencias. Después del diseño y elaboración de cada una de las guías, se desarrolló cada una de las conferencias en diferentes instalaciones y fincas del municipio de Granada, los productores conocieron cada uno de los equipos y el funcionamiento de los mismos para la obtención de licor de cacao. 


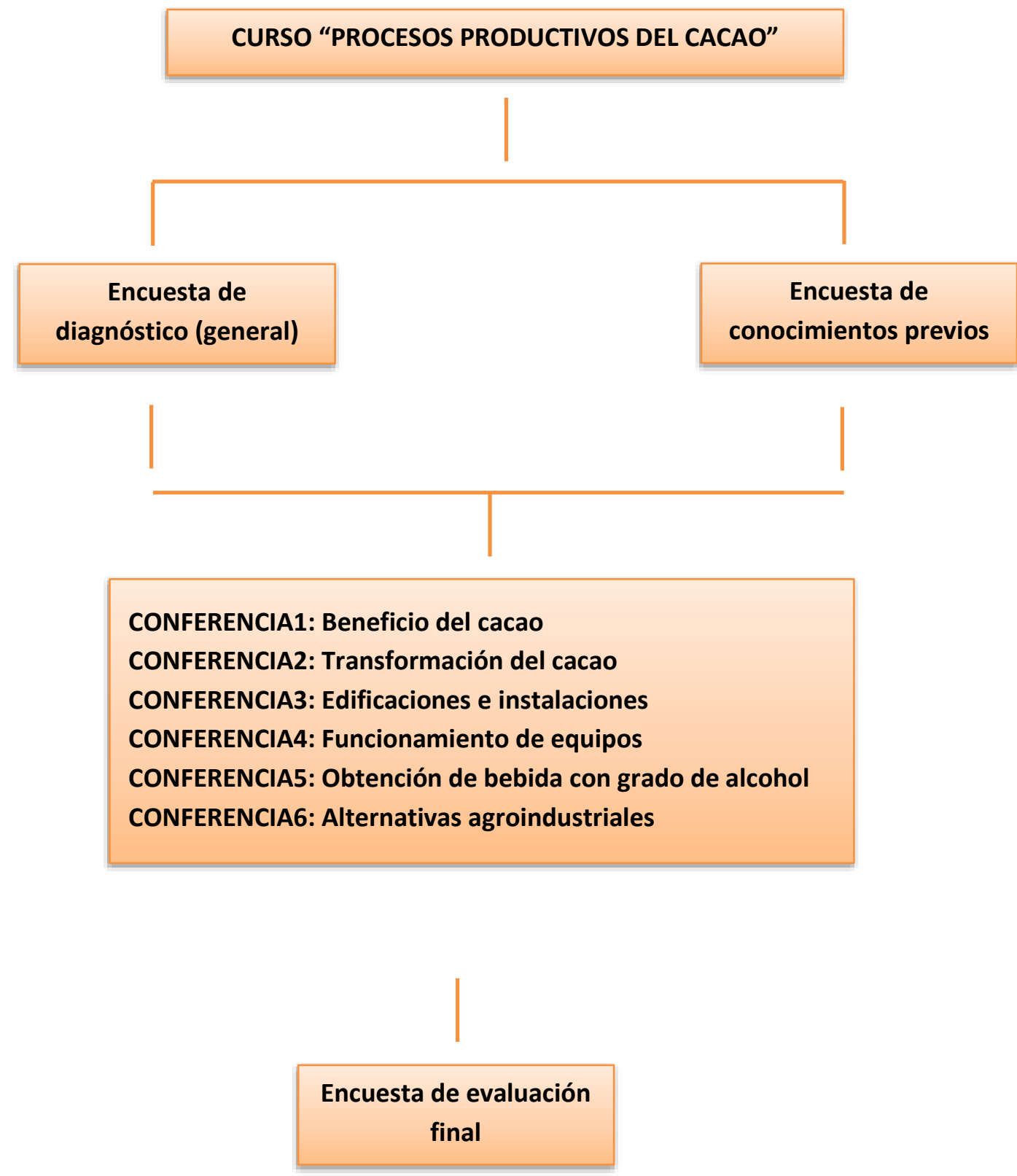

Figura 2. Actividades que se realizaron en este proyecto

En la tercera y última etapa, los productores diligenciaron la encuesta posterior que constaba de once preguntas en la cuales se preguntaba sobre los conocimientos que tenían los productores respecto al manejo del cultivo de Cacao en cuanto a beneficio, prácticas culturales, procesos de fermentación, métodos de secado, transformación y elaboración de derivados del cacao, esto fin de evaluar los conocimientos antes de tomar el curso "procesos productivos del cacao" para lo cual se aplicó un cuestionario de 11 preguntas y luego se aplicó el mismo cuestionario 
para comprar el aprendizaje adquirido después de haber participado en esta capacitación.

\section{Elaboración de guías}

Para el desarrollo de la conferencia procesos productivos del cacao se elaboraron seis guías, las cuales sirvieron de apoyo en cada conferencia presentada, compuesta por la descripción de la conferencia (temática, duración y tipo de guía), una breve introducción a la temática del día, objetivo de la conferencia, metodología a trabajar, información correspondiente a la conferencia y una actividad complementaria para reforzar el tema visto.

\section{RESULTADOS Y ANALISIS}

\section{Caracterización de la población objetivo}

La primera pregunta con referencia al tipo de población a la que pertenece se obtuvieron 10 víctimas, 22 mujeres, 21 campesinos y cero (0) afro descendientes, para un total de 50 participantes. La población a la que va dirigido el proyecto se encuentra en su mayoría mujeres con una presencia del $41 \%$ seguido de campesinos con un $40 \%$ y en un poco menos personas víctimas con un $19 \%$, (Figura 3). El 84\% de estos productores tienen cultivo de cacao, de los cuales el $62 \%$ han asistido a conferencias sobre el cultivo de cacao, frente a un $38 \%$ que nunca han tomado una capacitación al respecto.

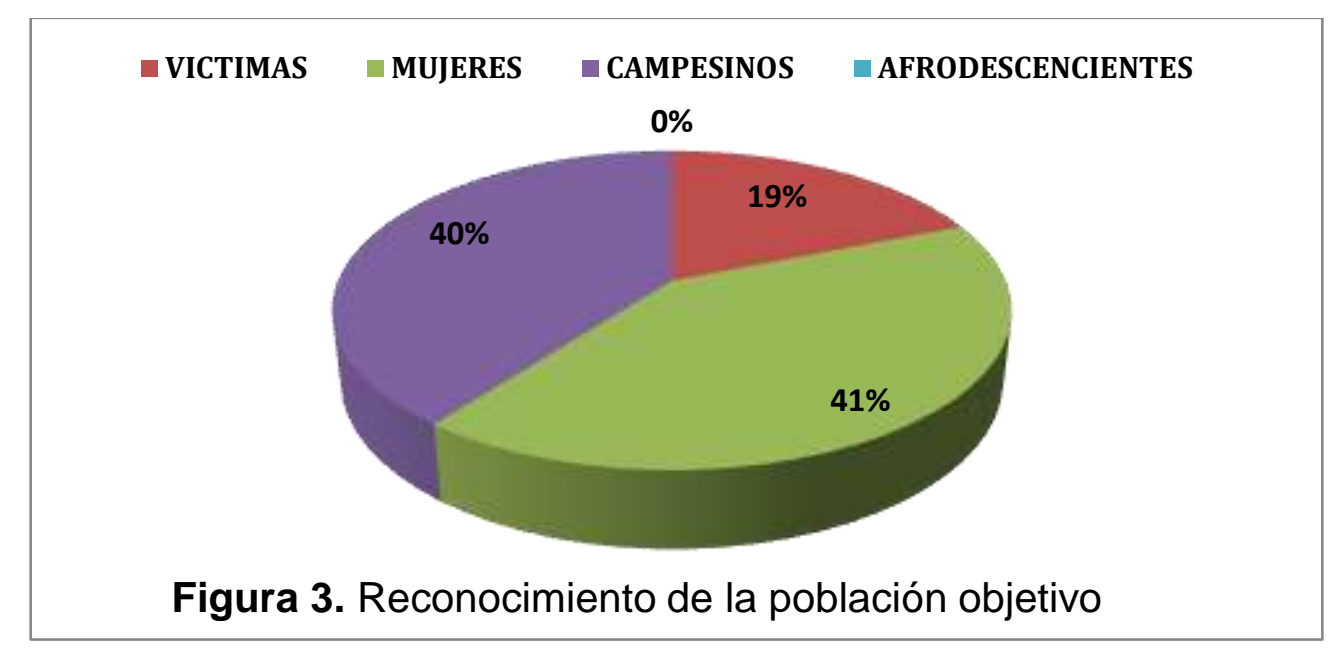


Se evidencia que en las fincas de Granada predomina el cultivo de cacao criollo con un $64 \%$ y en menos proporción el forastero y trinitario con un $18 \%$ cada uno, información suministrada por la plantación que dan en sus fincas y alrededores. (Figura 4).

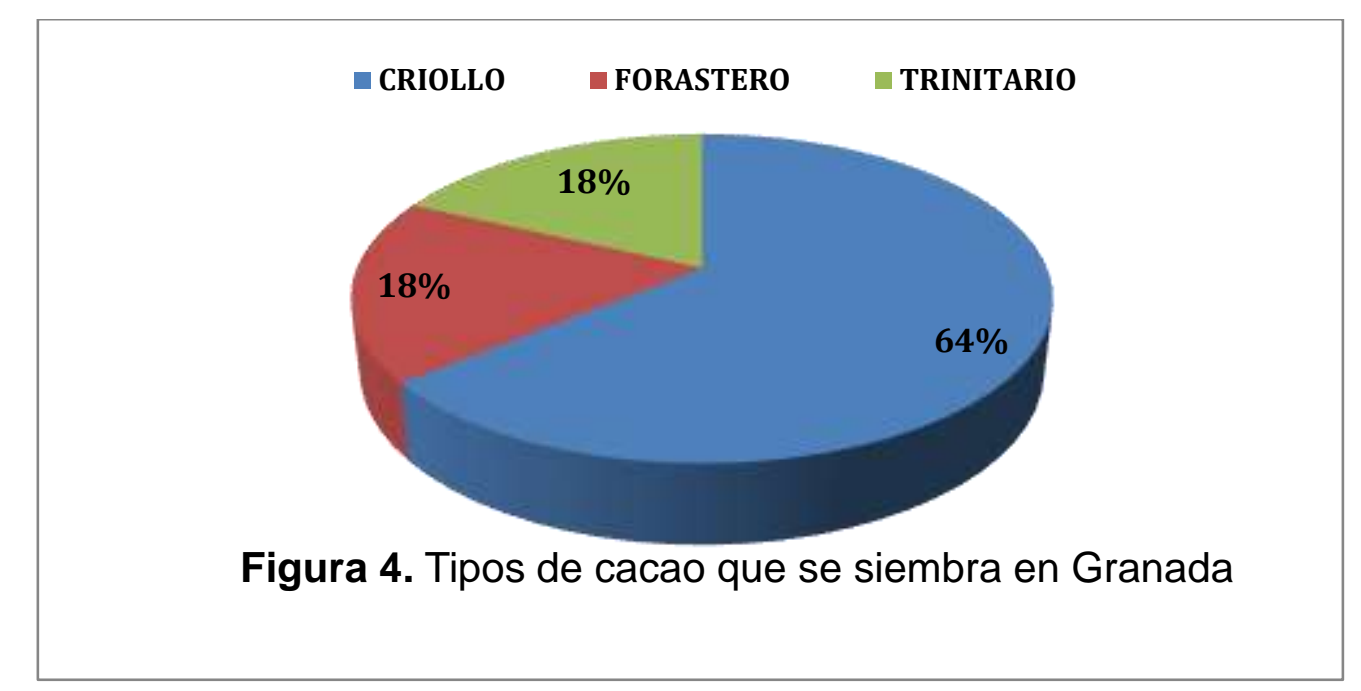

El $62 \%$ de los asistentes al curso procesos productivos de cacao han realizado fermentación del grano, mientras que el $38 \%$ no tienen conocimiento ni han realizado esta actividad. Con relación al tipo de fermentación que conocen, La fermentación de cacao de la cuál tienen mayor conocimiento los asistentes es la que se realiza en cajones con un $58 \%$, sin embargo, hay un $18 \%$ de personas que conocen los diferentes tipos de fermentación; mientras que el $14 \%$ no conoce ningún método (Figura 5). El 74\% de las personas que asistieron al curso ofrecido en este proyecto no han realizado transformación del cacao ni de forma artesanal y mucho menos tecnificada, sin embargo, el $26 \%$ de los asistentes está realizando productos derivados de cacao.

\section{Curso procesos productivos de cacao}

Este curso fue impartido en distintos lugares del municipio de Granada, donde asistieron y se certificaron 50 productores de cacao (22 son mujeres y 28 hombres) algunos pocos que ya le dan transformación, otros lo comercializan el cacao seco y una minoría que hasta ahora está iniciando en esta cadena, de los cuales 19 son 
víctimas del conflicto armado y 31 son campesinos. Se realizó el refuerzo de la cadena productiva desde su cultivo hasta la posible transformación.

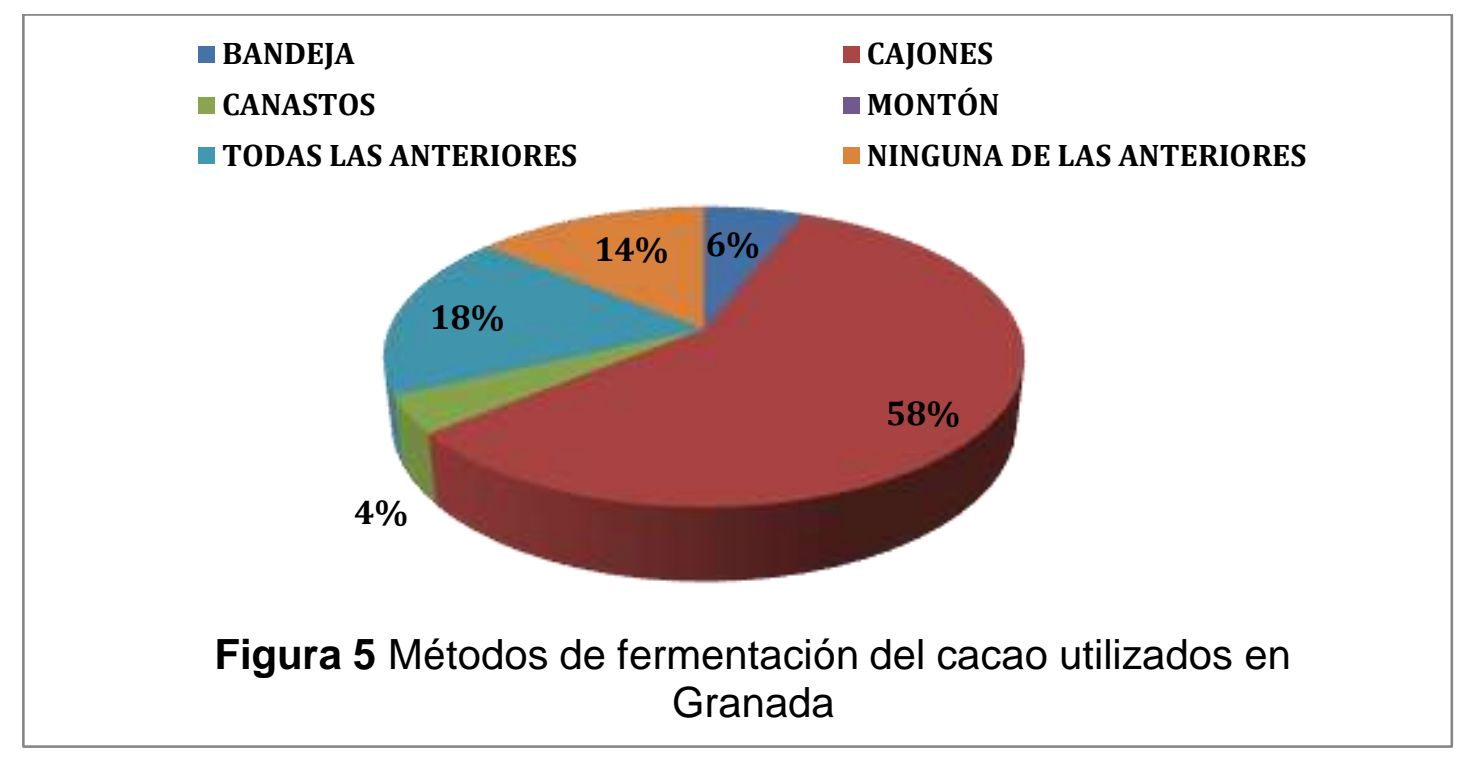

Beneficio del cacao: Se realizó en la finca "El refugio" ubicada en la vereda el delirio, para hablar sobre el cultivo, las características y condiciones óptimas de manejo, polinizadores, enfermedades, clones existentes en la región, la importancia de una buena poda, se realizó una actividad de campo donde todos los participantes mostraron gran interés en realizarla, conocer el cultivo de la finca visitada, reconocer su sistema, las enfermedades y recomendaciones para evitar el ataque de plagas. Se explicó sobre manejo de cosecha (características del grano), fermentación y secado, respecto a este tema se trató sobre el correcto proceso de estructuratemperatura-capacidad; humedad- temperatura- acidez- diseño de fermentador y secador, también se dieron a conocer algunos instrumentos para medir temperatura, humedad y acidez. Se fue a campo para ver el funcionamiento de los equipos, al igual, poner en práctica los conocimientos transmitidos, midiendo las condiciones de un cajón de fermentación, analizando si estaban bien o no y cuáles eran las medidas correctivas que se podrían aplicar (Figura 6).

\section{Transformación del cacao}

Se realizó en la finca trocha siete de punta brava, se dieron las pautas para realizar el proceso de forma artesanal, ingredientes y sus respectivas cantidades para la 
elaboración de chocolate de mesa con un $30 \%$ de cacao y otro con $100 \%$ de cacao. Se inició con un tostado de aproximadamente 30 minutos, se descascarilló se realizó manualmente separando en un recipiente el nib y en otro cascarilla (la cual es utilizada para infusiones), los nibs fueron llevados a un molino, posteriormente se agregó azúcar refinado y pulverizado, canela y clavos, lecitina de soya, se mezcló homogéneamente; esta mezcla paso por el molino, para formar una pasta para que la manteca de cacao se compacte con todos los ingredientes. Luego se procedió a vertir la pasta en los moldes y hacerle una vibración sobre mesa o superficie plana para poder eliminar cualquier burbuja de aire presente y luego se llevaron a refrigeración. Finalmente se obtuvo el chocolate de mesa, (Figuras 7 y 8 ).
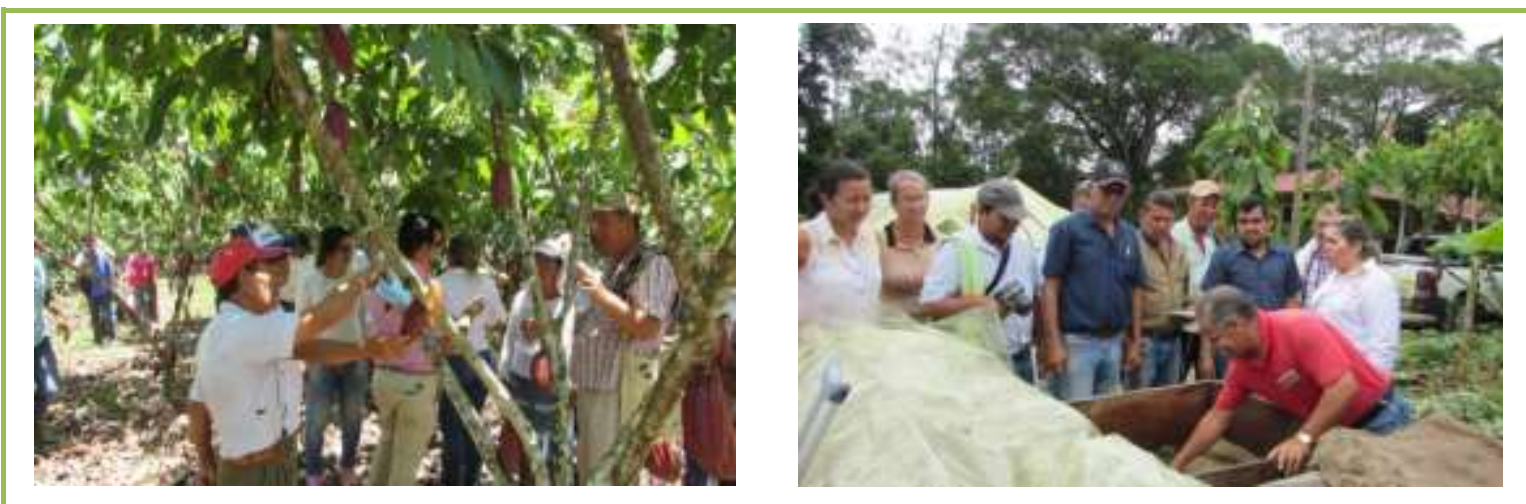

Figura 6. Práctica sobre variedades, plagas e insectos y proceso de fermentación del cacao.
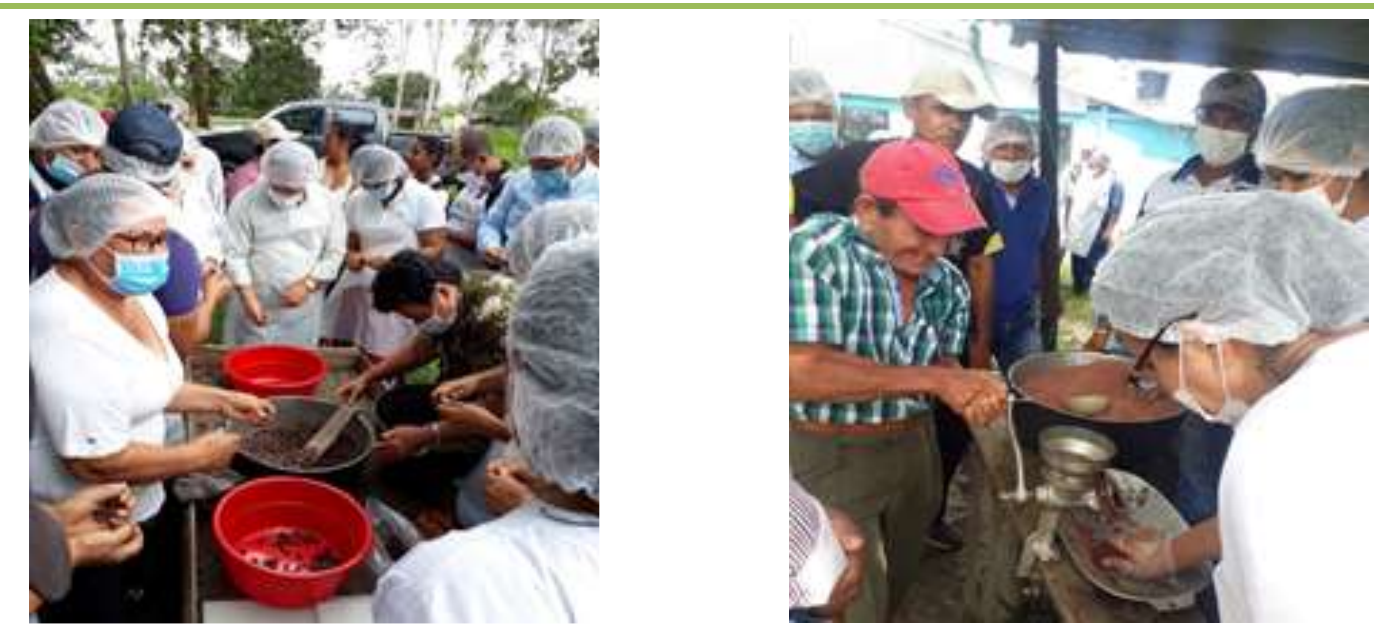

Figura 7. Práctica de transformación del cacao. 


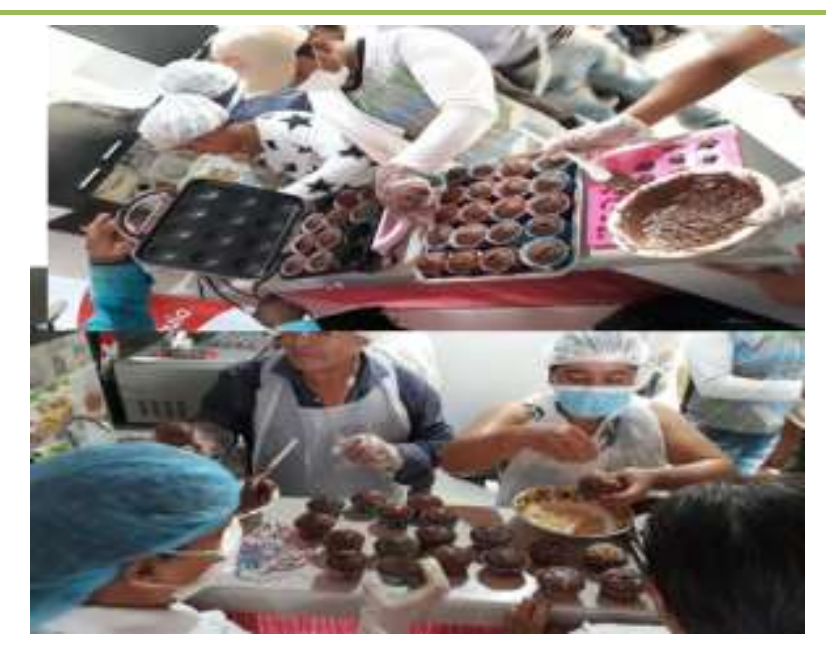

Figura 8. Elaboración de derivados del cacao.

\section{Alternativas de agroindustrialización}

Se explicó el funcionamiento de los equipos que se llevaron, se dio la fórmula de los cupcakes a preparar; Se armaron grupos de trabajo los cuales se iban a ir rotando para tener mejor control y participación de los asistentes, se incorpora poco a poco los ingredientes solidos previamente tamizados, luego los ingredientes líquidos cuando se obtiene una mezcla homogénea se retira y se agrega en la máquina para hacer cupcakes y se deja de 15 a 20 minutos, se dejaron enfriar para posteriormente proceder a decorarlos, Se atempera para la elaboración de los bombones los cuales iban a estar mezclados con maní o con arroz inflado, se controla la temperatura del atemperado $\left(42-44^{\circ} \mathrm{C}\right)$ se realizó la mezcla, se añadió en los moldes y se llevó a refrigerar para posteriormente degustarlos.

\section{Comparación de encuestas aplicadas antes y después de la conferencia "procesos productivos del cacao"}

El cuestionario que se realizó al inicio y final de las conferencias con el fin de evaluar los conocimientos que los participantes tenían acerca de la cadena productiva del cacao los cuales adquirieron o reforzaron en el tiempo transcurrido, se obtuvo que en relación a la temperatura óptima para el buen crecimiento de la planta de cacao al inicio contestaron: un participante marcó $15^{\circ} \mathrm{C}$, tres contestaron $20^{\circ} \mathrm{C}$, veintiocho 
respondieron que la respuesta correcta era $25^{\circ} \mathrm{C}$, diez que $35^{\circ} \mathrm{C}$ y finalmente ocho personas dijeron que $40^{\circ} \mathrm{C}$ era la temperatura óptima correcta. En la evaluación final 38 participantes respondieron $25^{\circ} \mathrm{C}, 9$ marcaron $35^{\circ} \mathrm{C}, 2$ que $40^{\circ} \mathrm{C}$ y 1 marcó $20^{\circ} \mathrm{C}$. En la evaluación de inicio el $56 \%$ de los participantes respondieron de forma correcta la pregunta indicando que tenían previo conocimiento, pero un $44 \%$ respondieron de forma incorrecta. En la evaluación final aumentó la cantidad de participantes que acertaron a la respuesta correcta con un $76 \%$ y una minoría respondió de forma incorrecta marcando 35,40 y $20^{\circ} \mathrm{C}$ con una participación de 18 , 4 y $2 \%$ respectivamente (Figura 9 ).

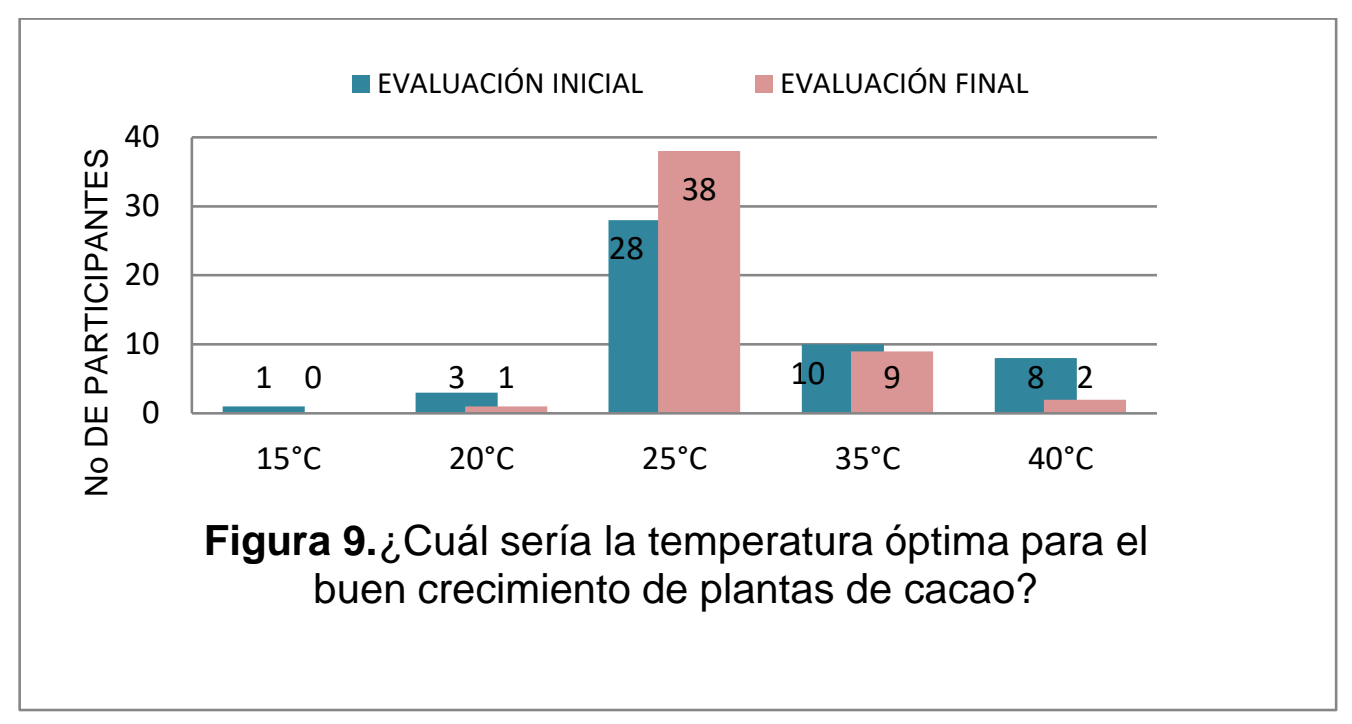

Referente a las evaluaciones sobre el conocimiento del cultivo de cacao y su transformación se exponen los resultados de las respuestas de dos preguntas de las 11 que se aplicaron antes y después de la capacitación; se puede observar que este curso aporto conocimiento a los productores de cacao (Figuras 9, 10 y 11). En relación al tiempo adecuado de fermentación del grano de cacao, en la evaluación de inicio cuatro personas respondieron de 3 a 4 días, treinta y cuatro de 6 a 7 días y doce personas respondieron que el tiempo adecuado era de 7 a 8 días, ya en la evaluación que se aplicó al final cuarenta y cuatro personas respondieron de 6 a 7 días, tres de 3 a 4 días y tres de 7 a 8 días. Un porcentaje significativo de los participantes con un $68 \%$ respondieron de forma adecuada donde el tiempo de fermentación indicado es de 6 a 7 días frente a un 32\% que respondieron de forma 
incorrecta en la evaluación de inicio, ya en la evaluación final aumentó la respuesta correcta de un $68 \%$ a un $88 \%$ (Figura 10 ).

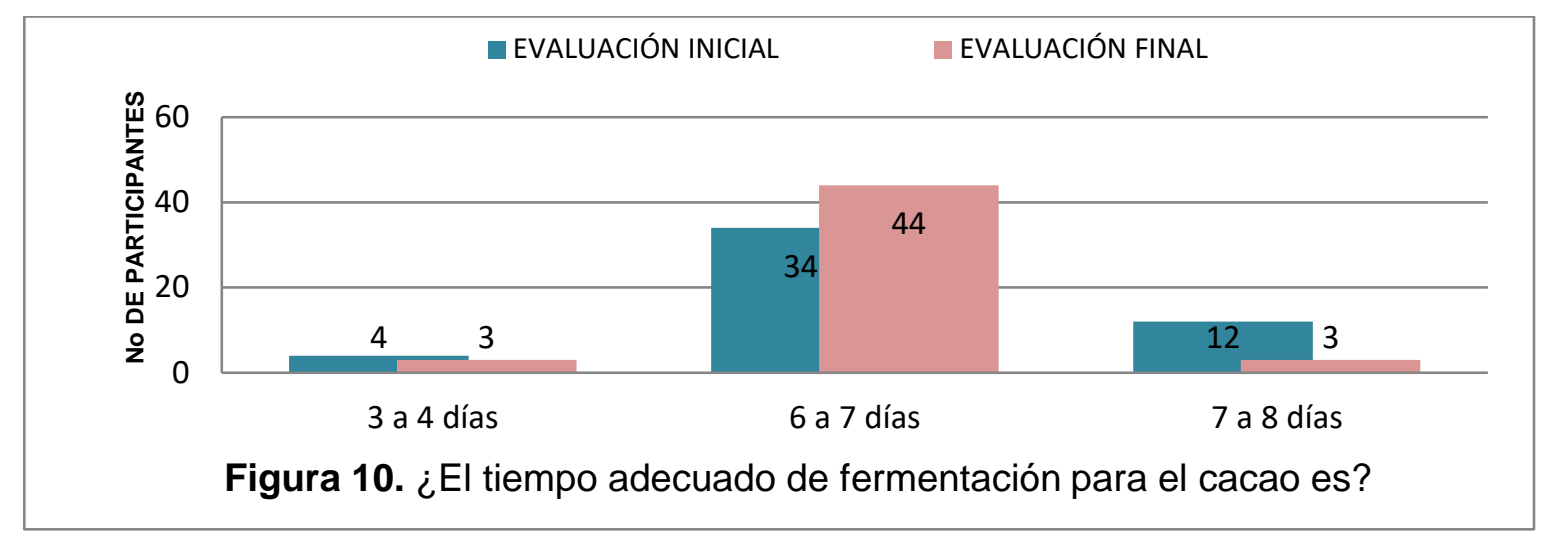

Sobre el conocimiento acerca del tiempo indicado para un correcto secado del grado de cacao a lo que en un inicio 14 personas respondieron que 3 días, 18 personas respondieron 5 días y 18 personas que 6 días. En la evaluación final 34 personas respondieron que 5 días era el tiempo ideal, 11 respondieron 6 días y 5 personas que 3 días. El tiempo ideal para el secado del grano de cacao es 5 días, en la encuesta de inició solo un $36 \%$ del $100 \%$ de los participantes acertaron con la respuesta correcta, pero en la encuesta final un $68 \%$ respondieron correctamente, aunque un $22 \%$ justificaron su respuesta en que en ocasiones se presentan fuertes lluvias lo que lleva al que el tiempo de secado se prolongue un poco (Figura 11).

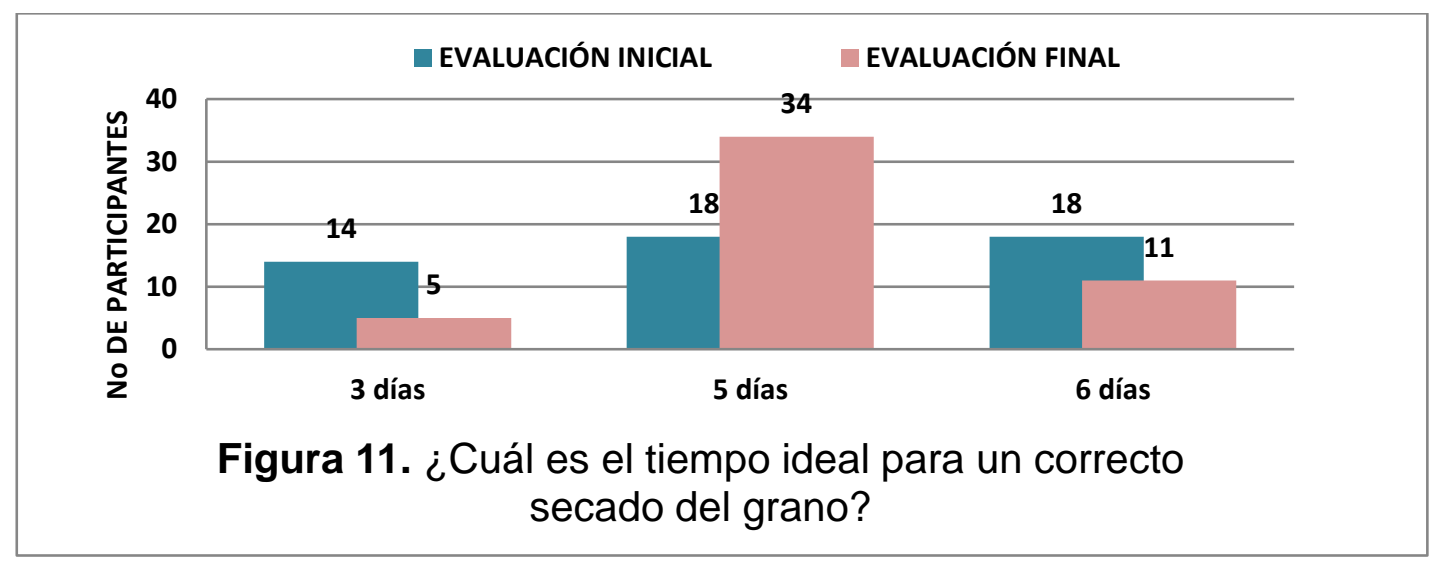

Con base a la encuesta inicial y final donde se buscó evaluar el conocimiento de los participantes se les efectuó una calificación de 1 a 5 dependiendo de las preguntas acertadas de las 11 del cuestionario. La calificación promedio del grupo al inicio fue 
de 1.91 indicando que la gran mayoría no tenía conocimiento o información suficiente sobre la cadena productiva del cacao lo cual los llevó a contestar de forma incorrecta algunas preguntas, teniendo en cuenta la calificación se profundizaron más las conferencias de tal manera que los participantes en su mayoría productores comprendieran la forma correcta de desarrollar un buen manejo del cultivo, beneficio y transformación del cacao, lo cual se logró y se vio reflejado en la evaluación final obteniendo una calificación promedio de 4.25 (Figura 12).

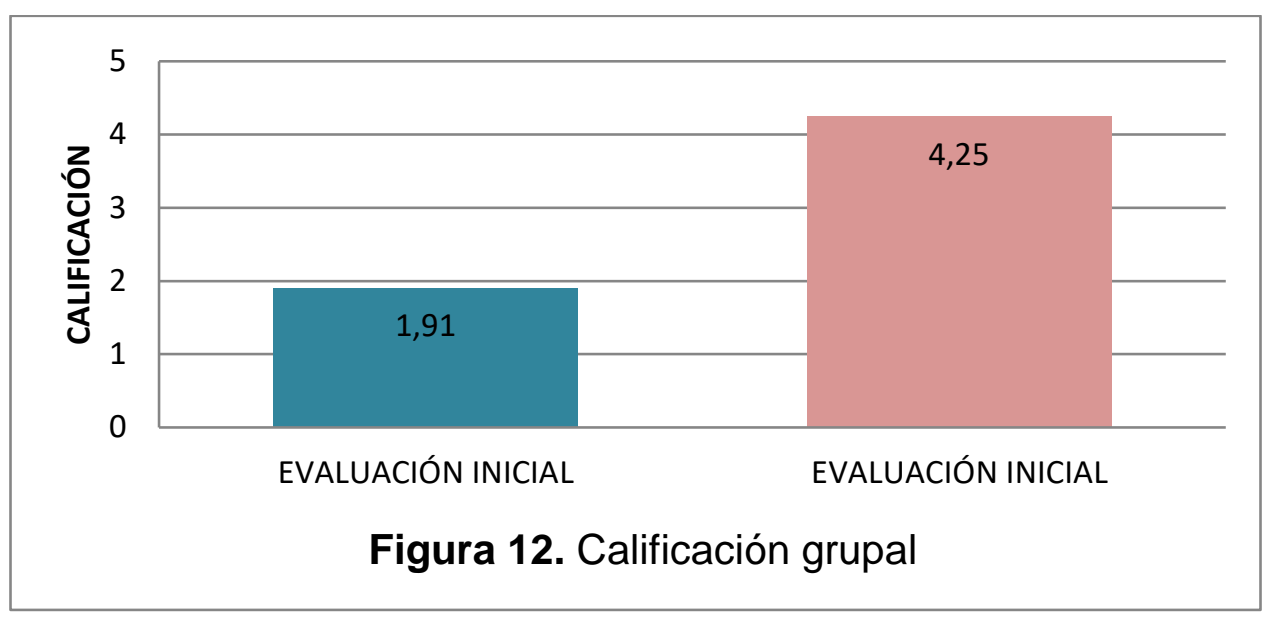

\section{CONCLUSIONES}

Se evidenció el aprendizaje adquirido por los participantes durante la conferencia reflejándose en la calificación general promedio que en un inicio fue de 1,91 y al final su calificación promedio ascendió a 4,25.

Las capacitaciones son una herramienta importante en el proceso de emprendimiento de pequeños y medianos productores de la región que buscan obtener mejores ingresos a partir no solo de la venta de materia prima si no dándole un valor agregado.

Los productores ahora con un panorama más despejado en este sector, dispuesto a buscar mejores alternativas para que su cultivo con mejores prácticas genere un cacao de mejor calidad, también reconocen la importancia de asociarse como pequeños y medianos productores de cacao ya que esto conlleva beneficios comunes, dimensionan poder generar negocio con lo aprendido al igual tienen en 
mira poder participar en más eventos similares que los forje como unos mejores agricultores cuidando y brindando armonía en su entorno.

Se dejó una semilla sembrada sobre el querer saber más sobre las diferentes cadenas productivas de la región, permitiendo así el ingreso de nuevos proyectos o entidades que alimenten el conocimiento y hagan acompañamiento a los productores; Partiendo de la conferencia ofrecida se podría abrir espacio en otros campos como lo es en temas relacionados a las buenas prácticas agrícolas, al marketing para la comercialización de los productos, capacitaciones en finanzas y emprendimiento para poder crear empresa.

La elaboración de este tipo de proyectos desde la academia hacía la comunidad son de gran aporte para el desarrollo de nuestro departamento siendo una forma efectiva de llegar aquellos hogares y lugares olvidados por muchos, pero donde se alberga una gran riqueza y contribuir al desarrollo de las regiones para avanzar hacía un mejor futuro en paz.

\section{REFERENCIAS BIBLIOGRÁFICAS}

1. Acosta A, Martínez C. 2017. Foto del artículo "Industria del cacao colombiano alcanza cifras históricas. En: Revista dinero. Octubre, 2017. Disponible en: https://www.dinero.com/edicion-impresa/pais/articulo/industria-del-cacaocolombiano-produce-record/251611

2. Arévalo A, González D, Maroto S, Delgado T, Montoya P. 2017. Manual técnico del cultivo de cacao: prácticas latinoamericanas. IICA. 143 p. Disponible en: http://creativecommons.org/licenses/by-sa/3.0/igo/ Creado a partir de la obra en www.iica.int

3. Argout, X; Salse, J; Aury, J; Guiltinan, M; Droc, G; Gouzy, J, Lanaud, C. 2011. the genome of theobroma cacao. Nature Genetics, 43 (2): 101-109.

4. Cediel, N; Donoso N; Hernandez, J; López, M; Herrera, P y Moreno, C. 2017. Empoderamiento de las mujeres rurales como gestoras de los Objetivos de Desarrollo Sostenible en el posconflicto colombiano. Equidad y Desarrollo, 28: 61 p.

Disponible

en: https://revistas.lasalle.edu.co/index.php/ed/article/view/4077/3226

5. Cely, L. 2017. Oferta productiva del cacao colombiano en el posconflicto. Estrategias para el aprovechamiento de oportunidades comerciales en el marco del acuerdo comercial Colombia-Unión Europea. Equidad Desarrollo, 28: 167-195. Disponible en: https://revistas.lasalle.edu.co/index.php/ed/article/view/4211/3232

6. Congreso de la República de Colombia (CRC). 2011. Ley 1448 DE 2011 (Junio 10). Por la cual se dictan medidas de atención, asistencia y reparación integral a las víctimas del conflicto armado interno y se dictan otras disposiciones. $75 \mathrm{p}$. 
Disponible en:

https://www.unidadvictimas.gov.co/sites/default/files/documentosbiblioteca/ley1448-de-2011.pdf

7. Cristancho, J; Guerrero, J, Romero J. 2017. Proyecto de ventajas comparativas reveladas del cacao Colombiano en la Alianza del Pacifico y oportunidad en el posconflicto. Bogotá, 60 p. Trabajo de grado (Profesional en negocios internacional). Universitaria Agustiniana. Facultad de ciencias económicas y administrativas.

Disponible

en: http://repositorio.uniagustiniana.edu.co/bitstream/handle/123456789/217/Guerrero Gordillo-JuanSebastian-2017.pdf?sequence=7\&isAllowed=y

8. Gómez R, Villanueva S, Henríquez M. 2019. Tendencia mundial en la elaboración de productos derivados del cacao. Revista INGENIERÍA UC, 26 (2). Disponible en: https://www.redalyc.org/jatsRepo/707/70760276010/html/index.html

9. Departamento Administrativo Nacional de Estadística (DANE). 2017. Cuentas trimestrales Colombia: Producto Interno Bruto (PIB) Cuarto Trimestre de 2017. Bogotá, Colombia. Disponible en: http://www.dane.gov.co/files/investigaciones/boletines/pib/bol PIB IVtrim17 ofert a demanda.pdf

10. Federación de Cacaoteros (FEDECACAO). 2018. El mundo quiere más cacao colombiano para este año. En: El Portafolio. Disponible en: https://www.portafolio.co/economia/el-mundo-quiere-mas-cacao-colombiano-paraeste-ano-516283

11. Gómez, J., Garcia, J. 2016. Violencia y pobreza en Colombia: Análisis de incidencia en Colombia a nivel departamental. En Econógrafos Escuela de Economía, № 95. Colombia, Disponible en: http://www.fce.unal.edu.co/centroeditorial/documentos/econografos-escuela-economia/1769-92-violencia-ypobreza-en-colombia-analisis-de-incidencia-en-colombia-a-niveldepartamental.html

12. Loureiro, G, Quintino R, Sodré R, Andrade G, Souza, S. 2017. Influencia de factores agroambientales sobre la calidad del clon de cacao (Theobroma cacao L.) PH-16 en la región cacaotera de Bahia, Brasil. Ecosistemas y recursos agropecuarios, 579-587. Disponible en: https://dx.doi.org/10.19136/era.a4n12.1274

13. Osorio, J, Berdugo, J; Coronado R, Zapata, Y; Quintero, C; Gallego, G; Yockteng, Roxana. 2017. Colombia es una fuente de diversidad genética del cacao según lo revelado por el análisis de la estructura de la población del banco de germoplasma de Theobroma cacao L. En: Frontiersea. Disponible en: https://www.frontiersin.org/articles/10.3389/fpls.2017.01994/full

14. Ramírez, H., Jaramillo, J. 2014. Uso potencial de agentes clarificantes y desinfectantes de origen natural para el tratamiento integral del agua caracterizado por pisos térmicos. Revista Ingeniería Solidaria, 10 (17): 139-151.

15. Rojas, F., Sacristán, E. 2013. Guía ambiental para el cultivo de cacao. Federación Nacional de Cacaoteros Ministerio de Agricultura y Desarrollo Rural, Ed 2. 2013. 127

p.

Disponible: https://www.fedecacao.com.co/portal/images/recourses/pub doctecnicos/fedecac ao-pub-doc 05B.pdf

16. Universidad de los Llanos (UNILLANOS). 2018 Informe de gestión Dirección General de Proyección Social primer semestre 2018, Villavicencio (Meta). 44 p. Disponible

en: http://proyeccionsocial.unillanos.edu.co/PSWEB\%203/Informes/IDG12018.pdf 